\title{
Practice Analysis of Road Traffic Crashes Accident of a City in China
}

\author{
Liwei $\mathrm{Hu}^{1, \mathrm{a}}$, Jian xiong ${ }^{2, \mathrm{~b}}$ \\ ${ }^{1}$ Faculty of Transportation Engineering, Kunming University of Science and Technology, Kunming \\ 650500, P.R.China; School of Transportation Science and Engineering, Harbin Institute of \\ Technology, Harbin 150090, P.R.China. \\ ${ }^{2}$ Faculty of Transportation Engineering, Kunming University of Science and Technology, Kunming \\ 650500, P.R.China. \\ aliweihukm@sina.com, bjebox@qq.com
}

Keywords: traffic accidents; crash features; contributory factor; crash type

\begin{abstract}
Many studies focused on the development of crash analysis approaches have resulted in aggregate practices and experiences to quantify the safety effects of human, geometric, traffic and environmental factors on the expected number of deaths, injuries, and/or property damage crashes at specific locations. Traffic crashes on roads are a major cause of road crashes in the metropolitan area of Xi'an. In an attempt to identify causes and consequences, reported traffic crashes for six years in Xi'an were analyzed using a sample of 2038 reports. The main types of information from such reports were extracted, coded, and statistically analyzed. Important results were obtained from frequency analyses as well as multiple contributory factors related to traffic crashes, including crash severity, time and location of occurrence, geometry of the road, AADT and $v / c$. This paper presents the results of such analyses and provides some recommendations to improve traffic safety and further studies to analyze potential crash locations.
\end{abstract}

\section{Introduction}

Worldwide deaths, disabilities, and injuries from road accidents, a major concern all over the world, have reached epidemic proportions. In 2002, more than 1.18 million people died in road crashes, and approximately fifteen million are injured annually (Source: World report on road traffic injury prevention: summary, 2004). In Europe, the sharp increase in accidents related to urban traffic costs more than two billion dollars during recent years. In Britain, around 3,500 people are killed each year and around 33,000 people are injured in road accidents. There were nearly 6,420,000 auto accidents reported to the police in the United States in 2005, and 2.9 million people were injured and 42,636 people killed, in which talking on a cell phone caused nearly $25 \%$ of accidents (Source: http://www.griefspeaks.com/id114.html). In New York City, specifically, the review of 2010 traffic accident database found: 1) 269 traffic deaths of all types and a 34 percent increase in auto fatalities; 2) 18 and 152 fatalities from bicycle accidents and pedestrian accidents, respectively (Source: http://www.lawfitz.com/new-york-city-traffic-accident-deaths-rise-slightly-in-2010). Nowadays, China has also witnessed a substantial rise in the number of traffic crashes, injuries and fatalities, especially since 1998 [1].

In response to these issues, various studies have recently examined aspects of motorcycle safety, in combination with available influencing variables and causes information. Typically, researchers employ statistical techniques (i.e., Poisson, negative binomial and regression models, etc.) in these types of studies [2]. Over the past years, numerous investigations have looked at the age and gender of drivers as risk factors, and younger and older drivers are more prone to be involved in a serious accident than median-aged ones [3]. Road geometric type, lightness, weather condition and other environmental factors (e.g., the time of the day, traffic volume, etc.) also play an important role when analyzing the aspects of accident crashes and injuries [4].

To provide a broad overview, numerous statistical researches have been conducted in order to understand the relations between influence factors and crash features via types of models [5]. Another class of studies utilize time series techniques for identifying the change of accident counts and 
accident rates at a given time, analyzing the effects of potential factors on accident occurrence, and developing a statistical model for forecasting future trends of crashes and injuries such as in references [6], and so on. Actually, each crash is a unique event that is caused or influenced by combinations of variables that may not even be observable [7]. Furthermore, reaching such conclusions that a set of variables can be identified as the causes of traffic crash is almost impossible.

This study follows an earlier work on the analysis of traffic accidents at intersections [8] and aims to identify the multiple crash features in Xi'an city. Several potential factors including district, human, vehicle, time, traffic volume, environmental and site factors are considered. Statistical analyses of multiple vehicle traffic accidents are conducted using the crash data in Xi'an city over the time of 2004 2009. The corresponding safety improvement measures are suggested so as to enhance the safety performance of road traffic and decrease the probability of crash occurrence in China's metropolitan regions.

\section{Crash Features}

Analyses were performed on the data collected from the General Administration of Transportation in the form of accident reports. The analyses included frequency, cross-classification tabulation, and construction of crash spot maps.

\section{Temporal-Spatial Patterns}

$\mathrm{Xi}$ 'an is at the top of list of cities with alarmingly high traffic crash fatality rates in China. A summary of crash type statistics for the year 2004 2009 for each district is presented in Table 1. More than $85 \%$ of crashes were vehicle collisions/crashes. About $53.8 \%$ of the total accidents occurred in the Yata district and Beilin district.

Table 1 Number of crashes and injuries by districts

\begin{tabular}{cccccccccc}
\hline \multirow{2}{*}{ Districts } & \multicolumn{4}{c}{ Crash Type } & \multicolumn{5}{c}{ Injuries } \\
\cline { 2 - 8 } & Collision & Running over & Overturning & Drugs & Total & Minor & Major & Total & \\
\hline Yanta & 587 & 24 & 32 & 19 & 662 & 70 & 36 & 105 & 38 \\
Beilin & 509 & 12 & 23 & 4 & 548 & 54 & 15 & 68 & 36 \\
Lianhu & 274 & 15 & 24 & 13 & 326 & 79 & 42 & 121 & 45 \\
Weiyang & 92 & 21 & 18 & 3 & 134 & 61 & 30 & 91 & 57 \\
Xincheng & 171 & 14 & 31 & 23 & 239 & 69 & 26 & 95 & 60 \\
Baqiao & 101 & 11 & 9 & 7 & 128 & 75 & 19 & 94 & 17 \\
Total & 1734 & 97 & 137 & 69 & 2038 & 407 & 167 & 574 & 253 \\
\hline
\end{tabular}

Around 318 fatalities occurred in the year 2009 with a decreasing trend. However, the overwhelmingly high proportion of younger drivers involved in traffic crashes has become a serious new concern. Over $60 \%$ of the fatalities for 2009 were in the age group of 18-40, though Xi' an had implemented new and stricter penalties under the new national traffic laws in May 2008 in order to minimize traffic crashes. The statistics show that the main cause of crashes is careless driving (e.g., mobile phone use while driving, fatigue, obsessive behavior, etc.) and speeding, which represent about $43 \%$ and $26 \%$ of the total crashes, respectively. The second main cause of crashes is drunk driving representing about $14 \%$. The rest of the reasons together represent about $17 \%$ of total crashes due to facilities, weather and other effects. Therefore, all but a few crashes can be blamed on careless driving, and other factors also play important roles.

Statistics also demonstrate that the safety situation in Xi'an was still serious as shown by the numbers of reported crashes during the years 2004 2009, which are presented in Table 2 . The number of crashes increased steadily during the years 2004 2006, and following this period began to decrease. Collisions represented the larger proportion of the total number of crashes. However, the crash rate was still at a high rate. The average number of crashes per day increased from 0.91 in 2004 to 1.06 in 
2006 and then decreased to 0.85 in 2009 . Crashes involving personal injuries fluctuated between a daily average of 0.15 to 0.4 and, fatal crashes which included at least one death occurred at a daily average of about 0.13 , which meant perhaps one life was lost weekly because of road crashes.

Table 2 Statistics of traffic crashes over the year 2004-2009

\begin{tabular}{ccccccc}
\hline Year & 2004 & 2005 & 2006 & 2007 & 2008 & 2009 \\
\hline Total number of crashes & 332 & 346 & 387 & 345 & 317 & 311 \\
Collision crashes & 270 & 284 & 324 & 291 & 262 & 259 \\
Crash of overrun/overturning/drugs/others & 62 & 61 & 64 & 54 & 55 & 52 \\
Total injuries & 113 & 73 & 102 & 146 & 86 & 54 \\
Minor injuries & 76 & 48 & 68 & 107 & 60 & 38 \\
Major injuries & 37 & 25 & 34 & 39 & 26 & 16 \\
Fatal crashes & 41 & 41 & 37 & 39 & 46 & 49 \\
Average no. of crashes per day & 0.91 & 0.95 & 1.06 & 0.95 & 0.87 & 0.85 \\
Average no. of injuries per day & 0.31 & 0.20 & 0.28 & 0.40 & 0.24 & 0.15 \\
Average no. of fatalities per day & 0.11 & 0.11 & 0.10 & 0.11 & 0.13 & 0.13 \\
\hline
\end{tabular}

\section{Frequency in Age and Weather}

Frequency analysis was performed for the following variables: time, day, weather, road surface condition, crash type, crash location, number of lanes, road type, car movement, crash type (general crash, rear end side collision), and severity.

Table 3 presents the injuries and deaths involved in crashes. Clearly, the number of male persons related to crashes was about three times of that of females and about a half of the injuries and deaths were drivers, which also confirmed the importance of educating the safe driving program. Moreover, the percentage of motorcyclists was up to $15.79 \%$, an alarming number among all the injuries and deaths. It was also noticed that $70.77 \%$ of injuries and deaths involved persons aging 25 60, especially those between 31 40, a group with higher driving risk. However, we found that the crash involvement rate of younger and older drivers was significant higher than that of medium aged ones.

Table 3 Injury and death categories involved in crashes

\begin{tabular}{cccccccc}
\hline Persons & Male & Female & Driver & Passenger & Pedestrian & Motorcycler & Bicycler \\
Percent $/ \%$ & 74.86 & 25.14 & 46.17 & 13.43 & 13.45 & 15.79 & 11.16 \\
\hline Age & $<16$ & $16-24$ & $25-30$ & $31-40$ & $41-50$ & $51-60$ & $>61$ \\
Percent $/ \%$ & 9.34 & 11.54 & 15.93 & 18.89 & 19.24 & 16.71 & 8.35 \\
\hline
\end{tabular}

Table 4 shows the effects of weather and brightness on crash occurrence. Obviously, the weather has a significant effect on crash occurrence and more than two third of observations occurred on bad weather days including cloudy, rainy, foggy, snowy, and heavily windy ones. Brightness also affects the frequency of crashes through affecting driving behavior of drivers, and we can see that as high as $17.94 \%$ of crashes during darkness, with street lamps.

Table 4 Crash distribution by weather and bright effect

\begin{tabular}{lcccccc}
\hline Weather & Sunny & Cloudy & Rainy & Foggy & Snowy & Heavy windy \\
Percent $\%$ & 31.73 & 13.79 & 17.38 & 11.17 & 17.48 & 8.45 \\
\hline \multirow{2}{*}{ Brightness } & Dawn & \multirow{2}{*}{ Daylight } & Twilight & Dark with street & Dark without street & Entrance and exit to urban \\
& & & & lamp & lamp & tunnel \\
Percent $\%$ & 1.33 & 63.74 & 6.15 & 17.94 & 9.97 & 0.87 \\
\hline
\end{tabular}

\section{Location Specification}

Crash distributions according to road type are shown in Table 5. It can be noticed that $33.46 \%$ of crashes were in arterial roads. Cumulative percentage shows that $45.09 \%$ of crashes occurred on expressways and arterials. This is because of high speeds and high traffic volumes on these two types 
of roads, and more frequent entrances and exits on such type of roads also contributed to the occurrence of crashes. The second highest incidence rate was in the sub-arterials category, with $19.72 \%$ of crashes occurring on them, due partially to the lack of effective safety facilities. Significantly, unsignalized intersections is a typical black spot prone to traffic crashes.

Table 5 Crash distribution by road type and crash location

\begin{tabular}{cccc}
\hline Road Type & Percent/\% & Position & Percent $/ \%$ \\
\hline Expressway & 11.63 & Roundabout & 2.49 \\
Arterial & 33.46 & Bridge & 0.65 \\
Sub-arterial & 19.72 & Tunnel & 0.87 \\
Branch and minor roads & 10.12 & Unsignalized intersection & 12.43 \\
Residential Street & 2.36 & Signalized intersection & 6.27 \\
\hline
\end{tabular}

Table 6 shows the cross tabulation of crash types and districts. It can be noticed from the results that car collision which had the highest percentage of crash occurrence had its highest percentage of $21.8 \%$ in Beilin district. This may be attributed to the high traffic volume and low speeds in these districts. The other types are noticed to have their highest percentage of occurrence in Weiyang and Baqiao. Unfortunately, truck-involved crashes made up a large percentage pattern in the crash reports, and this is particularly true for outlying or peripheral areas [10]. Roadside barriers, light poles, even trees and other stationary objects sometimes are prone to cause serious secondary collisions and severe injuries.

Table 6 Crash percentage in types on districts

\begin{tabular}{ccccccc}
\hline Crash type & Yanta & Beilin & Lianhu & Weiyang & Xincheng & Baqiao \\
\hline Car collision & 18.8 & 21.8 & 19 & 12.5 & 14.5 & 13.4 \\
Collision into stationary objects & 12.6 & 12.6 & 12.1 & 23.5 & 19.8 & 19.4 \\
Collision into pedestrians & 14.7 & 19.5 & 17.1 & 13.9 & 21.7 & 13.1 \\
Overturning & 18.9 & 15.1 & 22.5 & 13.3 & 11.9 & 18.3 \\
Falling down & 17.3 & 11.5 & 16.1 & 22.9 & 9.5 & 22.7 \\
Others & 17.7 & 19.5 & 13.2 & 13.9 & 22.6 & 13.1 \\
\hline
\end{tabular}

\section{Potential Causes}

As a result of reviewing the crash reports, crashes caused by sudden stop were observed statistically as the most frequent types, responsible for $32.3 \%$ of the total records. These usually occurred in traffic congestions where vehicles were moving slowly and traffic was mainly in stop-and-go situations, and drivers usually did not keep enough space between themselves and other vehicles [11]. Actually, traffic safety performance function (SPF), has a statistical relationship with congestion on urban freeways and observed safety, measured in the number of crashes over a unit of given time [crashes per kilometer per year (CPKPY)] and it varies with traffic exposure, measured in annual average daily traffic (AADT). Six years of data were used to analyze such effects on selected multi-type urban road segments. Figure 1 presents a pattern of total observed CPKPYs as the AADT increases for expressway and arterial segments. It should be pointed out that such a function for branch and minor streets was neglected due to a lack of crash records.

In Figure 1, traffic density at 34,000 AADT is a critical point and can be viewed as a critical density (point A), beyond which notably higher crash rates are observed with AADT changes, and the portion of the left of this critical density can thus be considered as a sub-critical zone, as AADT increasing from 8,000 to 34,000 induces the increase of traffic density by 3.5 times [from 5.7 pcu per kilometer per lane to $25.8 \mathrm{pcu} /(\mathrm{km} \cdot \mathrm{ln})]$, while traveling speed remains almost the same $(62 \mathrm{~km} / \mathrm{h}$ to $55 \mathrm{~km} / \mathrm{h})$. The portion to the right of point B can be viewed as a super-critical zone and the portion between 
sub-critical and super-critical densities can be viewed as a transitional zone, featuring an increase in CPKPY from 21 to 52, compared with 8-21 from point $\mathrm{C}$ to point $\mathrm{A}$. Further examination of SPF reflects that passing an AADT of 34,000, the number of crashes increases at a much faster rate with an increase in AADT.

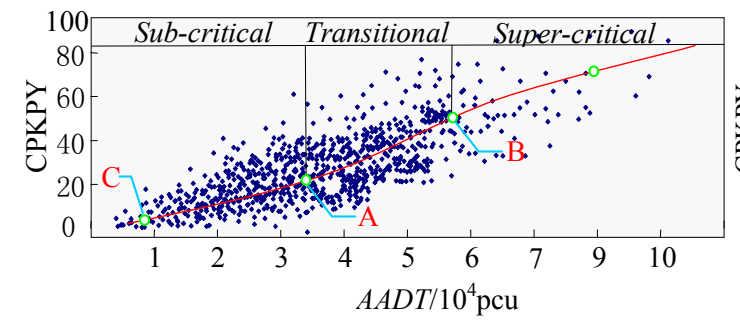

Figure 1 Statistical changes in segment crashes with AADT

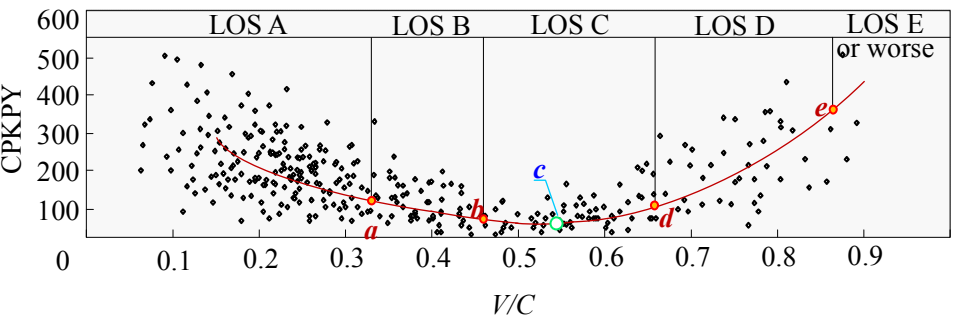

Figure 2 Statistical relation of crash rate and $v / c$

In actuality, the crash rate changes with traffic volume, and SPF reflects how these changes take place. Lower rates within equal SPF mean higher safety rather than higher rates. However, numerous research reports have shown that predictive models that use traffic volume as the only explanatory variable may not adequately characterize the crash process on freeway segments [12]. Functional forms that incorporate density and $\mathrm{v} / \mathrm{c}$ ratio offer a richer description of crashes occurring on these facilities, whether they are located in a rural or urban environment [13].

Figure 2 shows the relation between traffic crash rates measured in crashes per 100 million vehicle kilometers travelled and volume to capacity ratio (v/c). Obviously, the operation ranging 0.5 0.6 under LOS C meets the lowest level of crash rate and we yield the lowest 45 crashes with respective to moderate $\mathrm{v} / \mathrm{c}(0.54)$. Such a $\mathrm{V}$ regression mode also shows that free traffic flow usually brings serious rear-end or other types of crashes due to drivers' low vigilance of danger. Furthermore, more congested flow under LOS D or worse improves the likelihood of minor crashes.

Nevertheless, separate predictive models for single- and multi-vehicle crashes should be developed rather than one common model for all crash types [14]. Different variables (i.e., type of roads, barriers, etc.), for example, all have varying effects on the occurrence of crashes and statistical rates. Individual analysis may help find potential causes and effective measures to deal with the particular locations or long term safety improvement programs.

\section{Summary}

The presented study is designed to be a first step towards analyzing traffic crashes in Xi'an city. Crash features related to time, position, type of roads and type of crashes, etc., and exposure effects involving AADT and $\mathrm{v} / \mathrm{c}$ analyses provide many important types of information that are required by engineers and decision-makers to improve safety for the driving public in this western metropolis of China. Such analyses must be performed regularly (annually) for long-term traffic safety environment consideration [8].

During the review process of crash reports, some recommendations were suggested. Road geometry in Xi' an should be checked on the national level and improvements should be made at many locations that are not up to standards, for example improving sight distances at intersections and roundabouts; checking the appropriateness of (and redesign if necessary) the acceleration and deceleration lanes to and from arterials and sub-arterials [15], etc. Moreover, it is also recommended to increase awareness of traffic signals ahead by installing warning signs before intersections and to provide enough pedestrian crossings and overpasses, especially at heavy traffic intersections and commercial regions [16]. Sufficient traffic safety education is also necessary not only for drivers but also for voluntary participants (i.e., pedestrians, bicyclists, et al). For scientific research demand, crash messages need to be more accurately identified in order to construct more accurate crash database using available GPS technology [17]. 


\section{Acknowledgments}

This work was financially supported by the National Natural Science Foundation of China (Grant No. 51108137).

\section{References}

[1] D. D. Clarke, P. Ward, C. Bartle, et al, "Killer crashes: Fatal road traffic crashes in the UK", Accident Analysis and Prevention, 42(2), 2010, pp. 764-770.

[2] S. Mitra, "Spatial autocorrelation and Bayesian spatial statistical method for analyzing intersections Prone to injury crashes", Transportation Research Record, 2136, 2009, pp. 92-100.

[3] B. Falk, H. Montgomery, "Developing traffic safety interventions from conceptions of risks and accidents", Transportation Research Part F: Traffic Psychology and Behaviour, 10(5), 2007, pp. 414-427.

[4] T. F. Golob, W. W. Recker, "Relationships among urban freeway accidents, traffic flow, weather, and lighting conditions", Journal of Transportation Engineering, 129(4), 2003, pp. 342-353.

[5] X. S. Wang, A. A. Mohamed, P. A. Brady, "Crash estimation at signalized intersections significant factors and temporal effect”, Transportation Research Record, 1953, 2006, pp. 10-20.

[6] X. Ye, R. M. Pendyala, S. P. Washington, "A simultaneous equations model of crash frequency by collision type for rural intersections", Safety Science, 47(3), 2009, pp. 443-452.

[7] Y. G. Wang, K. M. Chen, Y. L. Pei, et al, "Integrating before and after crash features into measuring the effectiveness of intersection safety improvement project in Harbin", Transport, 26(1), 2011, pp. 112-121.

[8] H. Zhu, K. K. Dixon, S. Washington, et al, "Predicting single-vehicle fatal crashes for two-lane rural highways in Southeastern United States", Transportation Research Record, 2147, 2010, 88-96.

[9] X. P. Yan, M. Ma, Ming, H. L. Huang, et al, "Motor vehicle-bicycle crashes in Beijing: Irregular maneuvers, crash patterns, and injury severity", Accident Analysis and Prevention, 43(5), 2011, pp. $1751-1758$.

[10] K. Ratkeviciute, "Model for the Substantiation of road safety improvement measures on the roads of Lithuania", Baltic Journal of Road and Bridge Engineering, 5(2), 2010, pp. 116-123.

[11] I. Fi, J. Galuska, "Recommendations for new capacity values on freeways", Periodic Polytechnica: Civil Engineering, 54(2), 2010, pp. 127-136.

[12] J. Kononov, B. Bailey, B. K. Allery, "Relationships between safety and both congestion and number of lanes on urban freeways", Transportation Research Record, 2083, 2009, pp. 26-39.

[13] L. D. Zhong, X. D. Sun, Y. S. Chen, et al, "Research on the relationship between V/C and crash rate on freeway", Journal of Beijing University of Technology, 33(1), 2007, pp. 37-40.

[14] S. R. Geedipally, D. Lord, "Investigating the effect of modeling single-vehicle and multi-vehicle crashes separately on confidence intervals of Poisson-gamma models", Accident Analysis and Prevention, 42(4), 2010, pp. 1273-1282.

[15] A. Pande, M. Abdel-Aty, A. Das, "A classification tree based modeling approach for segment related crashes on multilane highways", Journal of Safety Research, 41(5), 2010, pp. 391-397.

[16] O. Prentkovskis, E. Sokolovskij, V. Bartulis, "Investigating traffic accidents: a collision of two motor vehicles", Transport, 25(2), 2010, pp. 105-115.

[17] S. S. Durduran, "A decision making system to automatic recognize of traffic accidents on the basis of a GIS platform”, Expert Systems with Applications, 37(12), 2010, pp. 7729-7736. 